\title{
Commonly used earthquake source models
}

\author{
Wenlong $\mathrm{Liu}^{1}$, Yucheng $\mathrm{Liu}^{2}$ \\ ${ }^{1}$ Shanghai Earthquake Administration, Shanghai 200062, China; \\ e-mail: wlliu_99@yahoo.com \\ ${ }^{2}$ Department of Mechanical Engineering, University of Louisiana, Lafayette, LA 70504, USA; \\ e-mail: yucheng.liu@louisiana.edu
}

\begin{abstract}
Several important earthquake source models that have been extensively used in seismological research and earthquake prediction are presented and discussed. A new fault source model is used to explain the earthquake focal mechanism solution and tectonic stress field, which play a crucial role in earthquake initiation and preparation. The elastodynamic-dislocation theory is demonstrated which provides the theoretical background of most earthquake source models. Important earthquake source models reviewed here include the double-force-couple point-source model, the circular-shear dislocation model, the finite moving-source model, the Brune model, and the spherical explosive source model.
\end{abstract}

Keywords: kinematic source model, focal mechanism solution, tectonic stress field, elastodynamic-dislocation theory, seismic rupture

\section{Introduction}

Tectonics-induced earthquakes are the most common and destructive earthquakes. They will occur anywhere within the earth where sufficient stored elastic strain energy is present to drive fracture propagation along a fault plane, and usually begin by an initial rupture at a point on the fault plane. Fault-model analysis is used to investigate a force system or dislocation applied on the fault plane that makes the displacement radiation at some distance consistent with the recorded seismic waves. The study of the fault source model can be traced back to the beginning of the $20^{\text {th }}$ century. Following the great 1906 San Francisco earthquake, Reid (1990) examined the displacement of the ground surface around the San Andreas Fault. From his observations he concluded that the earthquake must have been the result of elastic rebound of previously stored elastic strain energy in the rocks on either side of the fault, which is known as the famous 'elasticrebound theory'.

Kawasumi (1937) identified the quadrant distribution of the directions of P-wave first motion. However, this is not possible for teleseismic distances, and a method must be found to compensate for the effects on ray propagation of the variation of the velocity with respect to depth in the Earth. Byerly (1928) was the first to solve this problem by presenting a single force couple model to identify the fling motion of the two sides of a fault. An improved model, the double-force-couple model, was later developed by Honda (1957), which can better evaluate the seismic-wave radiation of point sources. Based on observation of the $S$-wave radiation patterns, it was found that the double-force-couple model is the best in 
describing the distribution pattern of P-wave radiation and S-wave polarizations.

It is of great help in studying earthquake source models to consider the earthquake source process as a rapid expansion of the rupture surfaces. The study of earthquake source models has two focuses. One is analysis of the kinematic model of the earthquake source, the objective of which is to find far-field seismicwave radiation, based on the temporal and spatial distribution of dislocations along the rupture surface. The second one is analysis of the dynamic model of the earthquake source, the objective of which is to find the motion of the medium on and near the rupture surface, based on the initial stress distribution, medium characteristics, and physical laws that govern the rupture process. The study of the dynamic model increases the insight into the initiation of the rupture and its process, while the study of the kinematic model provides an approach to inverse dynamic parameters of the earthquake source from seismograms.

In their study of the kinematic or dislocation model, Burridge \& Knopoff (1964) derived the integral of displacement caused by seismic dislocations under common conditions, using the Green's Function. A number of investigators studied the far-field displacement radiation in homogeneous, isotropic media caused by specifically distributed dislocations. Ben-Menachem (1961) studied the radiation of seismic surface-waves from finite moving sources; Savage (1965) investigated the shear dislocation circle; Haskell (1966) and Aki (1972) discovered the discontinuous propagation of ruptures; Wyss \& Brune (1967) studied a complex multi-rupture. In the study of the dynamic model, previous research interests focused on the propagation and stop of ruptures under the complex influences of friction forces, the non-elastic effect, and a heterogeneous initial stress. Aki \& Richards (1980) presented an approach to represent earthquake sources in terms of a static moment tensor. According to their theory, the usual approach towards source theory is to model inelastic processes due to faulting, explosions, etc. as causing a region of an elastic body (the source) to undergo a stress-free change of size and shape without alerting the elastic prop- erties of the region. If this change of size and shape is expressed as a strain, the seismic moment density tensor is defined by them as the product of such strain and the tensor of elastic moduli, based on which the displacement field can be determined. The static moment tensor model is now a general tool which is used to model and explain the seismic source.

The present contribution is organized as follows: section 2 reviews the double-force-couple point-source model and discusses the using of this model to explain the earthquake focal mechanism solution; section 3 explains the dislocation theory in terms of elastodynamics, where important equations of motion and their solutions are introduced; section 4 introduces several common kinematic source models such as the circular-shear dislocation model, the finite moving-source model, the Brune model, and the spherical explosive source model. Classical seismic-rupture models, including unilateral and bilateral ruptures, are also depicted in this section. Conclusions are drawn in section 5 .

\section{The double-force-couple point- source model}

The earthquake focal mechanism (P-wave first motion solution) deals with the two possible fault planes and orientations of principal stress axes and with dislocation directions obtained from the point-source single- and double-force-couple models. The single-forcecouple model assumes that the force system that acts on the earthquake source is equivalent to a single-force couple at the moment of

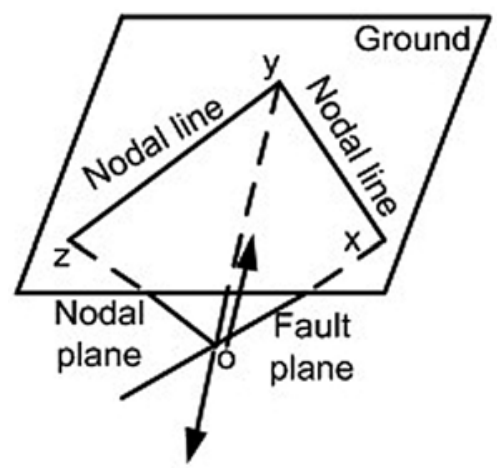

Fig. 1. Single-force-couple model. 
rupture, as shown in Figure 1. The origin point in this figure is the earthquake source. The $Y-X$ plane is the fault plane and the $X-Z$ plane is an auxiliary plane that is orthogonal to the fault plane and perpendicular to the rupture direction. Both planes are called 'nodal planes' and the intersection line of a nodal plane and the earth's surface is the nodal line. Based on this model, it can be derived that the fault plane and the auxiliary plane divide space into four quadrants; the directions of the P-wave first motion in neighbouring quadrants are opposite to each other, and the displacement of the P-wave on the nodal planes is zero. These conclusions are consistent with the observed distribution of the direction of P-wave first motion and radiation images.

The double-force-couple model assumes that the equivalent hypocentral-force system is a pair of force couples, the dislocation directions of which are perpendicular to each other, while their resultant couple is zero. The P-axis in Figure 2 is the principal compressive stress axis and the T-axis represents the principal tension-stress axis. The Z $\mathrm{Z}$-axis is the intersection line of the two nodal planes, and is called the 'neutral principal-stress axis' or 'zero axis'; it is marked using $\mathrm{B}$ or $\mathrm{N}$. The rupture direction is along the $\mathrm{Y}$-axis and its angles between the $\mathrm{P}$ - and T-axes are $45^{\circ}$. Both the single and the double-force-couple model yield the same farfield radiation, but different radiation images of the S-wave. Based on the single-force-couple model, a maximum amplitude of the S-wave is present in two directions, while the doubleforce-couple model shows the maximum amplitude in four directions. In practice, the maximum amplitude of the S-wave occurred in four

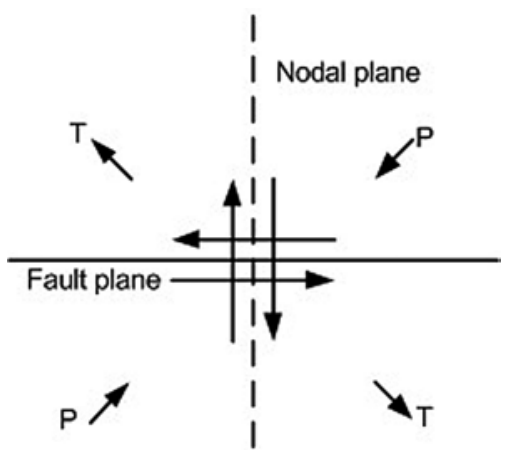

Fig. 2. Double-force-couple model. directions, which proves that the double-forcecouple model is more accurate in approximating the focal mechanism.

According to the double-force-couple model, the spatial orientations of the $\mathrm{P}_{-}, \mathrm{T}-, \mathrm{N}-$, and $\mathrm{Y}$-axes and of the two nodal planes can be determined from the distribution of the firstmotion direction of the P-wave. Nodal-plane solutions are usually found using the Wulf net; for nearby shocks, the solutions can be directly found on a plane. A corresponding accuracy should also be provided with the solutions, which is the maximum rotating angle about the nodal lines. When the angle is less than $5^{\circ}$, the accuracy is defined as class I; when the angle is between $5^{\circ}$ and $10^{\circ}$, it is defined as class II; and when the angle falls between $10^{\circ}$ and $15^{\circ}$, it is defined as class III. To find the nodalplane solutions, it is required that a number of seismic observatories evenly surround the epicentre. Alternatively, Li (1974) presented a way to find out the focal mechanism based on the amplitudes of the initial motion recorded by four observatories. Moreover, Li et al. (1973) developed a method to determine the nodal-plane solutions for small earthquakes based on the recorded first-motion directions of small and micro-earthquakes of a single observatory; they used this method to study the average stress field. The two nodal planes in

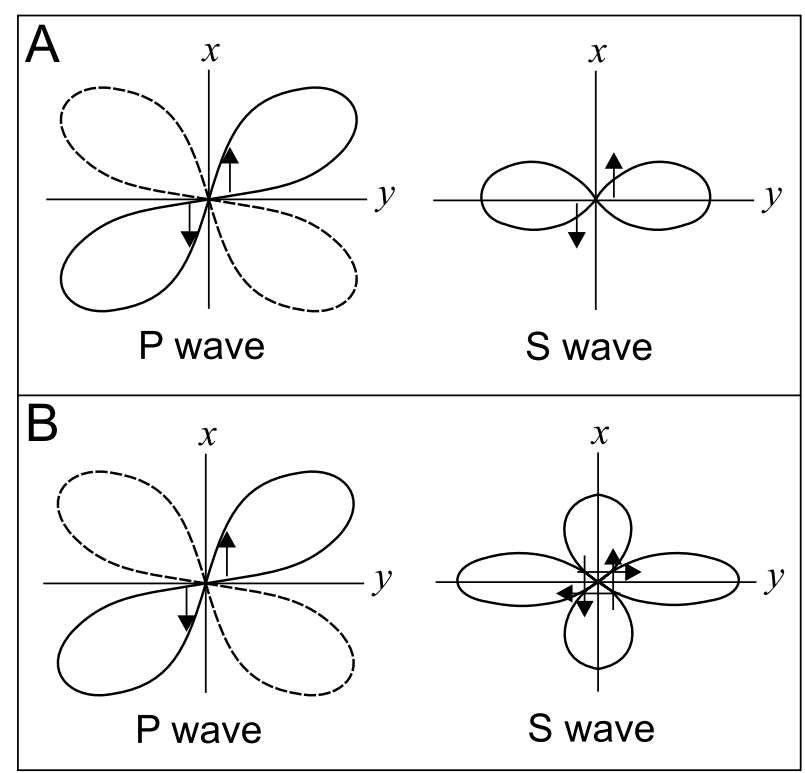

Fig. 3. Radiation images of P- and S-waves according to (A) the single-force-couple model and (B) the doubleforce-couple model. 
the double-force-couple model are perfectly symmetrical; therefore it is well known that the fault plane has to be determined through other approaches (distribution of seismic intensity, distribution of aftershocks, and field surveys into a seismogenic fault).

There are only few examples where the focal mechanism has been verified using the seismogenic fault. In a few examples, however, it was found that the seismogenic fault correlated well with the focal mechanism and that the directions of the fault plane determined using the two approaches are close (with differences of no more than $15^{\circ}$ ). The obtained fault plane of the 1974 Yongshan-Daguan earthquake with a magnitude of 7.11974 is a representative example (Table 1).

The obtained $\mathrm{P}_{-}, \mathrm{T}-$-, and $\mathrm{N}-$ axes are the principal-stress axes during earthquakes, which are not equal to the hypocentral principal-stress axes before the main shock. Under plane stress, the angle between the sliding surface and the $\mathrm{P}$-axis (the fault angle, $\mathrm{a}$ ) is related to the friction coefficient, $\mu$, as (following the CoulombNavier equation):

$$
\operatorname{tg}(2 \alpha)=1 / \mu
$$

According to Byerlee (1970), the granite under high confining pressure $\mu$ is

$$
\mathrm{M}=0.6+0.5 / \sigma_{\mathrm{n}}
$$

where $\sigma_{n}$ is the normal pressure on the sliding surface. It then can be estimated that a ranges from $25^{\circ}$ to $30^{\circ}$ in $\sim 10-30 \mathrm{~km}$ under the Earth's surface, so that the direction of the hypocentral principal stress before the main shock should be along $15^{\circ}$ to $20^{\circ}$ from the $\mathrm{P}$-axis to the fault plane.

In making integrated nodal-plane solutions for small earthquakes, different first-motion directions of the P-wave were usually found in the same quadrant. We define $\Psi$ as the contradiction ratio of the first-motion signal of the $\mathrm{P}$-wave, which equals the ratio between the number of contradictory signals and the total number of signals. Since one principle stress can cause two non-perpendicular fault ruptures, $\Psi$ caused by this principal stress will not approach zero, and can be calculated as:

$$
\Psi=\left(45^{\circ}-\mathrm{a}\right) / 90^{\circ}
$$

The lower bound of a can be evaluated from the maximum $\Psi$ value before the impending main shock. The estimated lower bound of $a$ is
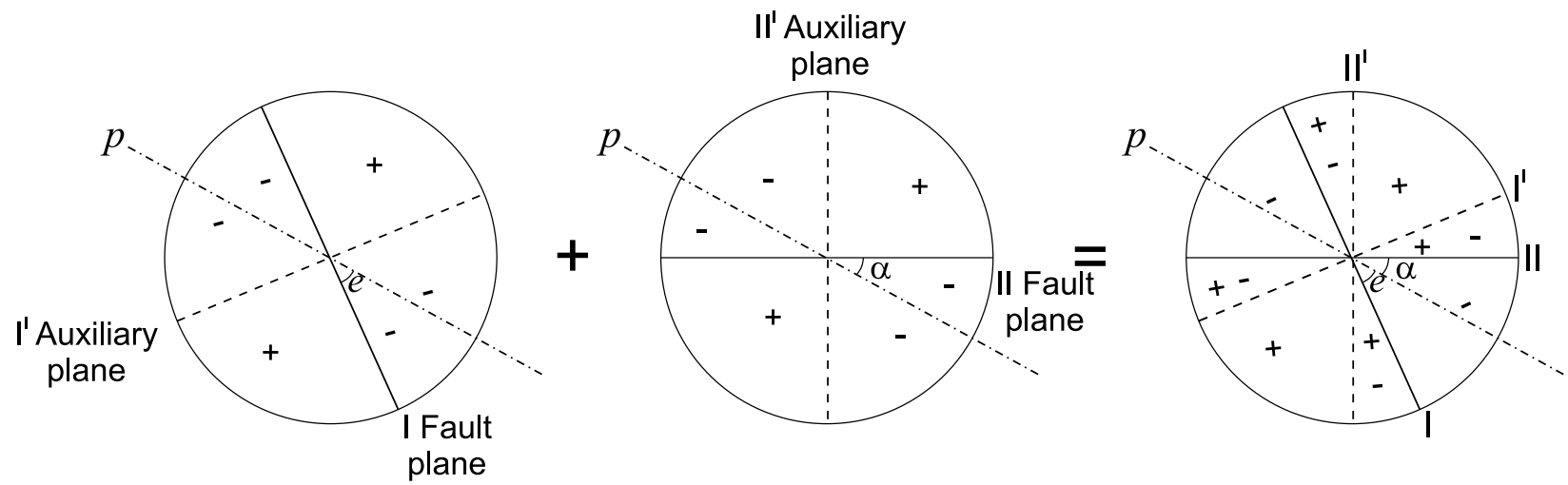

\begin{tabular}{|c|c|c|c|c|}
\hline & $\begin{array}{c}\text { P-wave first motion } \\
\text { solution }\end{array}$ & $\begin{array}{l}\text { Field surveys into the } \\
\text { jilipu fault }\end{array}$ & $\begin{array}{l}\text { Major axis of the } \\
\text { isoseismal curve }\end{array}$ & $\begin{array}{l}\text { Area in which the } \\
\text { after-shocks were } \\
\text { concentrated }\end{array}$ \\
\hline fault-plane direction & $34-214^{\circ}$ & $33-213^{\circ}$ & $35-215^{\circ}$ & $33^{\circ}$ \\
\hline fault-plane inclination & NE & NE & & NW \\
\hline
\end{tabular}

Fig. 4. Contradictory signals of the first motion, caused by $a \neq 45^{\circ}$.

Table 1. Fault plane of the Yongshan-Daguan earthquake (magnitude 7.1) on May 11, 1974. 
$27^{\circ}$ for the Beijing-Tianjin-Tangshan-Zhangjiakou area and $23^{\circ}$ for the Songpan-Pingwu area.

In practice, the principal axis of stress releasing was commonly used as the axis of principal stress at the hypocentre before the main shock, which is basically an application of the Coulomb-Navier law, neglecting the friction. To date, there is no good way to estimate the frictional coefficient $(\mu)$ of the individual rupture surface, so we have to assume $\mu=0$. This assumption does, fortunately, not affect the accuracy and precision of our analysis.

The stress field near the hypocentre before the earthquake may not be consistent with the regional or tectonic stress field. Under the influence of a large-range and long-term stable tectonic-stress field, a new stress field will be derived as a result of local tectonic movements and mutual interactions in the area near the hypocentre. The stress field near the hypocentre before the earthquake should be a superposition of the tectonic-stress field and the derived stress field. Thus, the principal direction of the individual earthquake cannot represent the direction of the tectonic-stress field. Generally, the direction of a local tectonic-stress filed can be approximated as the principal direction of the average stress related to the numerous earthquakes occurred in that the earthquakeaffected area. Especially the principal-stress directions of earthquakes with $M \geq 6$ are (except for strong aftershocks) quite stable and consistent in a large range, which fairly represent the direction of the tectonic-stress field.

It should be mentioned here that it was assumed in the double-force-couple point-source model, that the $\mathrm{P}_{-}, \mathrm{T}-$, and $\mathrm{N}$-axes are the principal-stress axes during earthquakes. However, McKenzie (1969) found that this assumption is true only if the fault plane is the plane of the maximum shear stress, and this is rarely true in the earth. Therefore, the accuracy and appropriateness of such an assumption need verification by future investigations.

\section{Dislocation theory based on elastodynamics}

\subsection{Integral expression of the solution of elastodynamic equations of motion}

Given a continuous linear elastic model with volume $\mathrm{V}$ and a situation subjected to different body forces, $\mathrm{f}_{\mathrm{i}}$ and $\mathrm{f}_{\mathrm{i}}$, assuming corresponding displacements, $u_{i}$ and $u_{i}{ }^{\prime}$, the equations of motion and Hooke's law can be written as

$$
\begin{gathered}
\sigma_{i j, j}+\rho f_{i}=\rho \ddot{u}_{i} \\
\sigma_{i j}=C_{i j k l} \cdot u_{k, l} \\
\sigma_{i j, j}^{\prime}+\rho f_{i}^{\prime}=\rho \ddot{u}_{i}^{\prime} \\
\sigma_{i j}^{\prime}=C_{i j k l} \cdot u_{k, l}^{\prime}
\end{gathered}
$$

where

$$
u_{k, l}=\frac{\partial u_{k}}{\partial x_{l}}, \quad \ddot{u}_{i}=\frac{\partial^{2} u_{i}}{\partial t^{2}}, \text { and } \sigma_{i j, j}=\sum_{j=1}^{3} \frac{\partial \sigma_{i j}}{\partial x_{j}}
$$

and $\mathrm{C}_{\mathrm{ijkl}}$ is the modulus of elasticity, which is symmetrical: $\mathrm{C}_{\mathrm{ijkl}}=\mathrm{C}_{\mathrm{klij}}$.

From Equations (4) through (8), the GreenVolterrra equation can be derived as:

$$
\begin{array}{r}
\int_{0}^{t_{1}} d t \oiint_{s}\left(u_{i}^{\prime} \sigma_{i j}-u_{i} \sigma_{i j}^{\prime}\right) n_{j} d s+\int_{0}^{t_{1}} d t \iiint_{v} \rho\left(u_{i}^{\prime} f_{i}-\right. \\
\left.-u_{i} f_{i}^{\prime}\right) d v+\left.\iiint_{v}\left(u_{i} \dot{u}_{i}^{\prime}-u_{i}^{\prime} \dot{u}_{i}\right) d v\right|_{t=0} ^{t=t_{1}} \text { (Eq. }
\end{array}
$$

where $S$ is the boundary surface of a volume, $\mathrm{V}$, and $\mathrm{n}_{\mathrm{j}}$ is direction cosines between ds and axis $x_{i}$.

Next, the Green tensor can be introduced to solve Equation (9). Consider the initial condition:

$$
u_{i}=\dot{u}_{i}=0 \text { when } t \leq t_{0}
$$


$S$ is a free surface so that we get for any vector $\mathrm{r}$ belonging to $\mathrm{S}$ :

$$
\sigma_{i j} \cdot n_{k}=0
$$

Parameter $\rho f_{i}$ is a unit-concentrated pulse force that acts on $r_{0}$ along $x_{k}$ at time $t_{0^{\prime}}$ which is:

$$
\begin{gathered}
\rho f_{i}=\delta_{i k} \cdot \delta\left(t-t_{0}\right) \cdot \delta\left(r-r_{0}\right), k= \\
=1,2,3
\end{gathered}
$$

We use $u_{i k}\left(r, r_{0}, t-t_{0}\right)$ to represent the solved displacement at $r$ along $x_{1}$ at time $t$, which is a second-order tensor, called the 'Green tensor'. Parameter $\delta$ in Equation (12) is the KroneckerDirac delta function, so that $\delta_{i j}=0$ when $\mathrm{i} \neq \mathrm{j}$ and $\delta_{i j}=1$ when $i=j$. Using $u_{i k}\left(r, r_{0}, t\right)$ and $u_{i l}(r$, $\left.r_{1}, t_{1}-t\right)$ and a relevant body force and stress to replace $\mathrm{u}_{\mathrm{i}^{\prime}}, \mathrm{f}_{\mathrm{i}^{\prime}} \sigma_{\mathrm{ij}}$, and $\mathrm{u}_{\mathrm{i}}{ }^{\prime}, f_{i}^{\prime}, \sigma_{i j}{ }^{\prime}$ in Equation (9), and applying initial and boundary conditions, we can obtain:

$$
U_{k l}\left(r_{0}, r_{l}, t_{l}\right)=u_{i k}\left(r_{l}, r_{0}, t_{l}\right)
$$

Equation (13) indicates that the displacement along $x_{k}$, which is caused by the unit-concentrated pulse force acting at $r_{1}$ along $x_{1}$, equals the displacement along $x_{1}$ which is caused by the force acting at $r_{1}$ along $x_{k}$. Such a relationship is known as the reciprocity theorem.

Substituting the unit-concentrated pulse force and the corresponding Green tensor from Equation (12) into Equation (9) as $f_{i}{ }^{\prime}$ and $u_{i}{ }^{\prime}$, and applying initial conditions, the integral expression of the solution of elastodynamic equations of motion can be solved by using the reciprocity theorem as:

$$
\begin{gathered}
u_{k}\left(\vec{r}_{0}, t_{0}\right)=\int_{0}^{t_{1}} d t \oiint_{s} u_{k l}\left(\vec{r}_{0}, \vec{r}, t-t_{0}\right) \cdot \sigma_{i j}\left(u_{i}\right) \cdot n_{j} \cdot \\
\cdot d s-\int_{0}^{t_{1}} d t \oiint_{s} u_{i}(\vec{r}, t) \cdot \sigma_{i j}^{\prime}\left(u_{k l}\right) \cdot n_{j} \cdot d s+ \\
+\int_{0}^{t_{1}} d t \iiint_{v} u_{k l}\left(\vec{r}_{0}, \vec{r}, t-t_{0}\right) \cdot f_{i} \cdot d v \text { (Eq. } 14
\end{gathered}
$$

where the first item represents the effect of the stress $\sigma_{i j}$ along the boundary surface, $S$, on the displacement field; the second item illustrates the influence of the displacement, $u_{i}$ of $S$ on the displacement filed; and the third item describes the contribution of the body force, $\mathrm{f}_{\mathrm{i}^{\prime}}$ to that field. It can be deduced from the above example, that solutions of most equations of motion can be simply expressed by using of the Green tensor, $\mathrm{u}_{\mathrm{k}{ }^{\prime}}$ even though the tensor itself might be difficult to formulate for general distributions of $\rho(r)$ and $C_{i j k l}(r)$ in a given volume, V.

\subsection{Theory}

The displacement caused by a dislocation along a plane $\Sigma$ which occurred in a given volume $\mathrm{V}$ at time $\mathrm{t}=0$ can be approximately solved without considering the body force. Assuming the initial condition:

$$
u_{i}=\dot{u}_{i}=0 \text { at } t=0
$$

the stress is continuous when passing through $\Sigma$, and $S$ is a stress-free surface:

$$
\begin{aligned}
u_{i}^{+}-u_{i}^{-} & =\Delta u_{i}(r, t), \sigma_{i j} \cdot n_{j}=0, r \\
& \text { belongs to } \Sigma
\end{aligned}
$$

The displacement field created by the dislocation then can be solved as:

$$
\begin{gathered}
u_{k}\left(\vec{r}_{0}, t_{0}\right)=\int_{0}^{t_{1}} d t \iint_{\Sigma} \Delta u_{i}(\vec{r}, t) . \\
\cdot C_{i j l m} \frac{\partial u_{k l}\left(\vec{r}_{0}, \vec{r}, t_{0}-t\right)}{\partial x_{m}} \cdot n_{j} \cdot d s
\end{gathered}
$$

Equation (17) is the integral expression of the displacement caused by dislocation. Here we assume that the plane, $\Sigma$, is enclosed with a closed curve surface, $S$ (Fig. 5) and that the contribution of the surface element, ds, to the total displacement during interval $\mathrm{dt}$ is: 


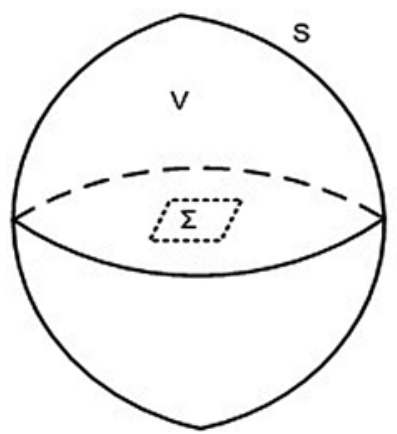

Fig. 5. A closed-curve surface, $S$, that envelops $\Sigma$.

$$
d u_{k}=\Delta u_{i} \cdot C_{i j l m} \cdot n_{j} \cdot d s \cdot d t \cdot \frac{\partial u_{k l}}{\partial x_{m}}
$$

It van be deduced from Equation (18) that the displacement field of radiation $\mathrm{du}_{\mathrm{k}}$ caused by the element dislocation $\Delta u_{i}$ per unit surface and generated per unit time, equals the displacement field created by the resultant couple of nine couples which act at the point source. The influence of each couple is $\Delta u_{i} \cdot C_{i j l m} n_{i}$ and the displacement field of the radiation caused by $\Sigma$ is the same as the field created from such point sources which continuously distribute on the plane, $\Sigma$, in media without ruptures.

In seismic studies, we are more concerned about shear dislocation, in which the direction of the dislocation $\left(e_{i}\right)$ is perpendicular to the orientation of rupture plane $\Sigma\left(n_{i}\right): e_{i} n_{i}=0$. Assuming that the medium is isotropic homogeneous, then $\mathrm{C}_{\mathrm{ij} / \mathrm{m}}$ becomes:

$$
C_{i j l m}=\lambda \sigma_{i j} \cdot \delta_{l m}+\mu\left(\delta_{i l} \cdot \delta_{j m}+\delta_{i m} \cdot \delta_{j l}\right)
$$

Substituting Equations (19) and (18) into Equation (17), the displacement radiation of the shear dislocation can be obtained as:

$$
\begin{array}{r}
u_{k}(\vec{r}, t)=\iint_{\Sigma} \Delta u \cdot \mu \cdot e_{i} \cdot n_{j} d s \cdot\left(\frac{\partial u_{k i}\left(\vec{r}, \vec{r}_{0}, t-t_{0}\right)}{\partial x_{j}}+\right. \\
\left.+\frac{\partial u_{k j}\left(\vec{r}, \vec{r}_{0}, t-t_{0}\right)}{\partial x_{i}}\right) \ldots i \neq j \quad \text { (Eq. 20) }
\end{array}
$$

This equation shows that the displacement radiation of the shear dislocation is equivalent

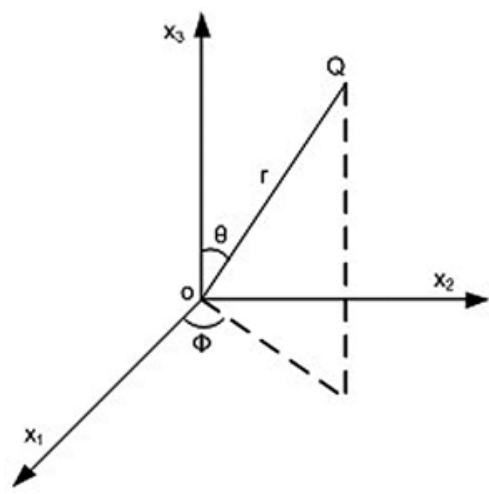

Fig. 6. Coordinate system composed of $\mathrm{O}$ (position where the dislocation locates), $X_{1}$ (direction of dislocation), and $X_{3}$ (orientation of the dislocation plane).

to the seismic-wave radiation caused by the double-force-couple that acts at point sources.

A coordinate system can be established, the original point of which is assumed to be the position of a dislocation element (Fig. 6); the dislocation is then expressed as:

$$
\Delta \vec{u}(\vec{r}, t)=\left.\Delta \vec{u}\right|_{\vec{r}=0} \cdot G(t)
$$

where $G(t)$ outlines the increase of the dislocation, which is also known as the hypocentralsource time function. The Green tensor, $\mathrm{u}_{\mathrm{k} \mathrm{r}^{\prime}}$ in Equation (21) is the displacement caused by the concentrated force in the isotropic, homogeneous medium. Substituting (21) into (20) and neglecting the item $\mathrm{O}\left(1 / \mathrm{r}^{2}\right)$, the displacement field of radiation for a shear dislocation element in an isotropic, homogeneous medium can be obtained as:

$$
d u_{j}=\frac{d m_{0}}{4 \pi \rho c^{3} r} \Re_{j} \times \dot{G}\left(t-\frac{r}{c}\right)
$$

of which the Fourier's spectrum is:

$$
d \hat{u}_{j}=\frac{d m_{0}}{4 \pi \rho c^{3} r} \Re_{j} \cdot i \omega \cdot \hat{G}(\omega) \cdot e^{-i \omega \frac{r}{c}}
$$

In Equation (22), $\rho$ is the density, c can be a or $\beta$, which are the velocities of the P- and $S$-wave, respectively. The hypocentral radiant intensity is:

$$
d m_{0}=\mu \cdot \Delta \bar{u} \cdot d S
$$


where $\mathrm{m}_{0}$ is the seismic moment, $\Delta \bar{u}$ denotes the average dislocation, and $\mathrm{dS}$ is the area of the dislocation surface element. $\dot{G}\left(t-\frac{r}{c}\right)$ in Equation (22) represents the influence of the hypocentral-source time function; $1 / \mathrm{r}$ represents the geometric diffusion; $\mathfrak{R}_{j}$ means the radiation pattern, which reflects the effect of the position of the observatory. In the coordinate system shown in Figure 6, we have:

$$
\left\{\begin{array}{l}
\Re_{r}=\sin 2 \theta \cdot \cos \varphi \\
\Re_{\vartheta}=\cos 2 \theta \cdot \cos \varphi \\
\mathfrak{R}_{\varphi}=-\cos \theta \cdot \sin \varphi
\end{array}\right.
$$
have:

When $c=\alpha, j=r ; c=\beta, j=\theta$ and $\varphi$, we can

$$
\begin{gathered}
\left\{\begin{array}{l}
u_{\alpha}=u_{r} \\
u_{\beta}=\sqrt{u_{\theta}^{2}+u_{\varphi}^{2}}
\end{array}\right. \\
\left\{\begin{array}{l}
\mathfrak{R}_{\alpha}=\mathfrak{R}_{r} \\
\mathfrak{R}_{\beta}=\sqrt{\mathfrak{R}_{\theta}^{2}+\mathfrak{R}_{\varphi}^{2}}
\end{array}\right.
\end{gathered}
$$

\section{Frequently used kinematic source models}

\subsection{The circular-shear dislocation model}

In order to obtain far-field displacement radiation, we need first to assume a geometric shape of the rupture surface, determine the dissemination mode of ruptures, and estimate the distribution of dislocation along the rupture surface and the rising shape of the dislocation (hypocentral-source time function), and so on. Different assumptions need be taken in the case of different objects and study objectives; different hypocentral dislocation models are thus established.

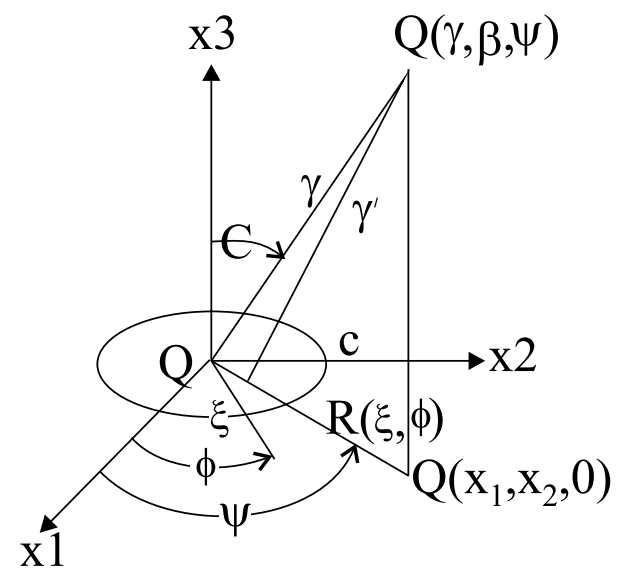

Fig. 7. The circular-shear dislocation model.

The circular-shear dislocation model (Fig. 7) is suitable for middle and small earthquakes, and assumes (Chen et al., 1976) that (1) the rupture surface is circular; (2) shear fracture starts from the centre of the circle and propagates radially at a constant velocity, $\mathrm{v}_{\mathrm{f}}$; (3) the dislocation is a Volterra dislocation, which is an evenly-distributed dislocation, which is represented as:

$$
\Delta \vec{u}(\vec{\xi}, t)=\Delta \vec{u} \cdot G\left(t-\frac{\vec{\xi}}{v_{f}}\right)
$$

where $\xi$ is the distance from a dislocation element to the centre of the circle, and $G(t)$ is defined as

$$
\begin{gathered}
G(t)=\frac{1}{2}\left(1-\cos \frac{\pi t}{T_{s}}\right) \cdot\left[H(t)-H\left(t-T_{s}\right)\right]+ \\
+H\left(t-T_{s}\right)
\end{gathered}
$$

which is the hypocentral-source time function and $\mathrm{H}(\mathrm{t})$ is the Heaviside function and when $\mathrm{t} \geq$ $0, \mathrm{H}(\mathrm{t})=1$, while for $\mathrm{t}<0, \mathrm{H}(\mathrm{t})=0$. In Equation (29), $\mathrm{T}_{\mathrm{s}}$ is the hypocentral time constant and $\mathrm{T}_{\mathrm{s}}$ $=4 \mathrm{a} / 7 \beta_{\mathrm{s}}$, where $\mathrm{a}$ is the radius of the dislocation circle and $\beta_{\mathrm{s}}$ is the velocity of the S-wave at the hypocentre.

Integrate Equation (22) over the above circular rupture plane and its far-field displacement radiation can be obtained as: 


$$
u_{j}(r, t)=\frac{m_{0}}{4 \pi \rho c^{3} r} \Re_{j} \dot{G}(t) * F_{c}\left(t-\frac{r}{c}\right)
$$

In Equation (30), “*” means convolution, and $F_{c}(t)$ depends on the area of the dislocation surface, $\mathrm{S}$, and on $\mathrm{v}_{\mathrm{f}}$ as:

$$
\begin{aligned}
& F_{c}(t)=\frac{v_{f}^{2} \cdot t}{S}\left\{\frac{2 \pi}{\left(1-\varepsilon_{c}^{2}\right)^{3 / 2}}\left[H(t)-H\left(t-t_{2 c}\right)\right]-\right. \\
& -\left[\frac{2}{1-\varepsilon_{c}^{2}} \cdot \frac{\sqrt{\left(t-t_{1 c}\right)\left(t_{2 c}-t\right)}}{t}+\frac{4}{\left(1-\varepsilon_{c}^{2}\right)^{3 / 2}} \cdot t^{-1}\right. \\
& \sqrt{\left.\left.\frac{\left(1+\varepsilon_{c}\right)\left(t-t_{1 c}\right)}{\left(1-\varepsilon_{c}\right)\left(t_{2 c}-t\right)}\right] \cdot\left[H\left(t-t_{1 c}\right)-H\left(t_{2 c}-t\right)\right]\right\}}
\end{aligned}
$$

where:

$$
\left\{\begin{array}{l}
t_{1 c}=\frac{a}{v_{f}}\left(1-\varepsilon_{c}\right) \\
t_{2 c}=\frac{a}{v_{f}}\left(1+\varepsilon_{c}\right) \\
\varepsilon_{c}=\frac{v_{f}}{c} \sin \theta
\end{array}\right.
$$

If $G(t)$ is the Heaviside function $H(t), F_{c}(t)$ reflects the waveform of the body wave displacement (Fig. 8). Thus it can be found that the semi-period of the first motion is proportional to the hypocentral radius and that the logarithm of the first-motion amplitude is proportional to the logarithm of that radius.

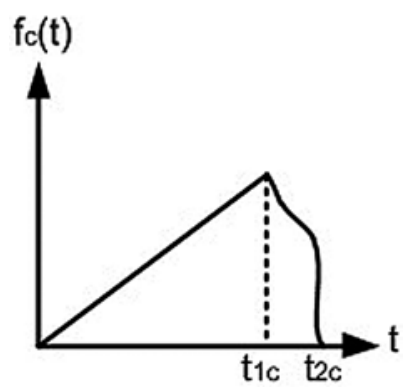

Fig. 8. The waveform of the body wave displacement.
The far-field displacement radiation in the frequency domain is:

$$
\hat{u}_{j}(r, \omega)=\frac{m_{0}}{4 \pi \rho c^{3} r} \Re_{j} \cdot i \omega \hat{G}(\omega) \cdot e^{-i \frac{\omega r}{c}} \cdot \hat{F}_{C}(\omega)
$$

In this equation:

$$
\left\{\begin{array}{l}
\left\{\begin{array}{l}
\hat{F}_{c}(\omega)=\frac{i v_{f}}{\omega S} \int_{0}^{2 \pi}\left[\frac{a}{q_{c}(\psi)} \cdot e^{-i \frac{\omega q_{c}(\psi) a}{v_{f}}}+\right. \\
q_{c}(\psi)=1-\varepsilon_{c} \cos \psi \\
\varepsilon_{c}=\frac{v_{f}}{c} \cos \theta \\
\left.+\frac{v_{f}}{i \omega q_{c}^{2}(\psi)}\left(e^{-i \frac{\omega q_{c}(\psi) a}{v_{f}}}-1\right)\right] d \psi
\end{array}\right.
\end{array}\right.
$$

and for a very low $\varpi$ we have:

$$
\left.\hat{u}_{j}(\omega)\right|_{\omega \rightarrow 0}=\frac{m_{0}}{4 \pi \rho c^{3} r} \Re_{j}
$$

It can be deduced from the above equations that the seismic moment, $\mathrm{m}_{0}$, can be determined from the low-frequency horizontal line and that the corner frequency, $f_{c}$ (a frequency corresponding to the intersection between the low-frequency horizontal line and the high-frequency asymptote) is inversely proportional to the hypocentral radius.

\subsection{The finite moving-source model}

\subsubsection{Unilateral rupture}

The finite moving-source model assumes that the rupture surface is a rectangular plane and that the rupture propagates along one or both sides at a constant velocity. This model 
is good for approximating large earthquakes within the lithosphere. The reason is that the rupture propagation distances along different directions are not roughly equal, due to the limited depth of the lithosphere. For the sake of simplification, all dislocations are assumed to be Volterra dislocations.

Unilateral rupture (Fig. 9) assumes that the rupture originates from one side and propagates unilaterally at a constant velocity, $\mathrm{v}_{\mathrm{f}^{\prime}}$ of which the far-field displacement is:

$u_{j}(r, t)=\frac{m_{0}}{4 \pi \rho c^{3} r} \Re_{j} * F(c) * \Omega_{c}\left(t-\frac{r}{c}\right)($ Eq. 36)

where:

$$
\left\{\begin{array}{l}
m_{0}=\mu \cdot \Delta \bar{u} \cdot W \cdot L \\
F(c)=\frac{1}{1-\frac{v_{f}}{c} \cos \psi} \\
\Omega_{c}(t)=\frac{v_{f}}{\Delta \bar{u} L}\left[\Delta u(t)-\Delta u\left(t-\frac{L}{V_{f}} F(c)\right)\right]
\end{array}\right.
$$

$\mathrm{W}$ and $\mathrm{L}$ are the width and the length of the rupture plane, respectively. The half period of the first motion is:

$$
T_{u}=L\left(\frac{1}{v_{f}}-\frac{\cos \psi}{c}\right)
$$

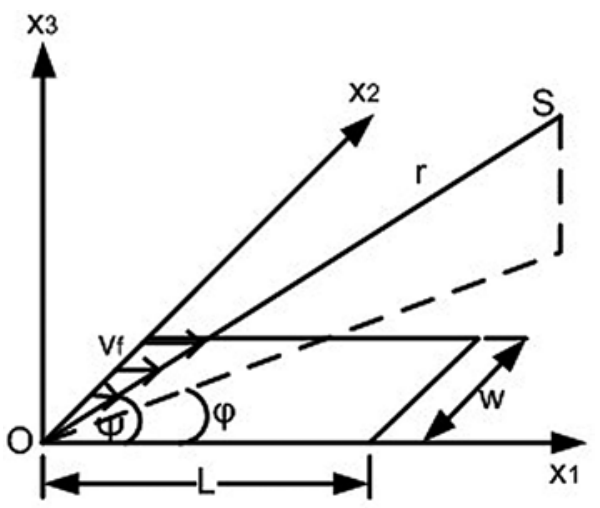

Fig. 9. Unilateral rupture.
The spectrum of the far-field displacement in the frequency domain is:

$$
\left\{\begin{array}{l}
\hat{u}_{j}(r, \omega)=\frac{m_{0}}{4 \pi \rho c^{3} r} \Re_{j} \cdot F(c) \cdot \Omega_{c}(\omega) \\
\Omega_{c}(\omega)=i \omega \cdot \hat{G}(\omega) \cdot e^{-i \frac{\omega r}{c}-i X} \cdot \frac{\sin X}{X} \\
X=\frac{\omega L}{2}\left(\frac{1}{v_{f}}-\frac{\cos \psi}{c}\right)
\end{array}\right.
$$

In this equation, $\sin X / X$ is called the 'directional factor'. A series of nodal points are found in the amplitude spectrum, the locations of which are determined based on $\mathrm{x}=\mathrm{nп}(\mathrm{n}=$ $1,2,3 \ldots)$. The corresponding frequency of the nodal points $f_{n}$ is:

$$
f_{n}=\frac{n}{L\left(\frac{1}{v_{f}}-\frac{\cos \psi}{c}\right)}
$$

It can be deduced from this equation that $1 /$ $\mathrm{f}_{1}=\mathrm{T}_{\mathrm{u}}$. The fault plane can be determined using either $\mathrm{f}_{1}$ or $\mathrm{T}_{\mathrm{u}^{\prime}}$ as well as its $\mathrm{L}$ and $\mathrm{v}_{\mathrm{f}}$.

\subsubsection{Bilateral rupture}

The bilateral rupture model (Fig. 10) assumes (Lin et al., 1979) that (1) the rupture starts from a position on the rectangular rupture surface and propagates over distances $\mathrm{L}_{0}$ and $\mathrm{L}_{\Pi}$ along two opposite directions; (2) the propagation velocities on the two sides are $\mathrm{v}_{\mathrm{f} 0}=\mathrm{v}_{\mathrm{fr}}=\mathrm{v}_{\mathrm{f}}$ of which the far-field displacement is:

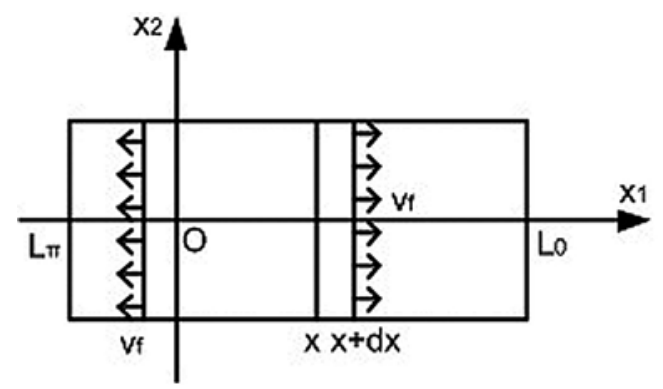

Fig. 10. Bilateral rupture. 


$$
\begin{gathered}
u_{j}(r, t)=\frac{m_{0}}{4 \pi \rho c^{3} r} \Re_{j} \cdot \sum_{i=0}^{n}\left\{\frac{v_{f} \cdot F_{i}(c)}{\Delta \bar{u} L}\right. \\
{\left[\Delta u\left(t-\frac{r}{c}\right)-\Delta u\left(t-\frac{r}{c}-\frac{L_{i}}{v_{f} \cdot F_{i}(c)}\right)\right]}
\end{gathered}
$$

where:

$$
\left\{\begin{array}{l}
m_{0}=\mu \cdot \Delta \bar{u} \cdot W \cdot L \\
L=L_{0}+L_{\pi} \\
F_{0}=\frac{1}{1-\frac{v_{f}}{c} \cos \psi} \\
F_{\pi}=\frac{1}{1+\frac{v_{f}}{c} \cos \psi}
\end{array}\right.
$$

The spectrum of the far-field displacement in the frequency domain is:

$$
\left\{\begin{array}{l}
\hat{u}_{j}(r, \omega)=\frac{m_{0}}{4 \pi \rho c^{3} r} \Re_{j} \cdot i \omega \cdot \hat{G}(\omega) \cdot e^{i \frac{\omega r}{c}} \\
L=L_{0}+L_{\pi} \\
X_{0}=\frac{\omega L_{0}}{2}\left(\frac{1}{v_{f}}-\frac{\cos \psi}{c}\right) \\
X_{\pi}=\frac{\omega L_{\pi}}{2}\left(\frac{1}{v_{f}}+\frac{\cos \psi}{c}\right)
\end{array}\right\}
$$

A special bilateral rupture where $\mathrm{L}_{0}=\mathrm{L}_{\pi}$ is called a 'symmetric bilateral rupture', if $\Delta u(t)=\Delta \bar{u} \cdot H(t)$, the half period of its first

motion $T_{u}=\frac{L}{2}\left(\frac{1}{v_{f}}-\frac{|\cos \psi|}{c}\right)$, of which the spectral curve, $\lg \left|\hat{u}_{j}(\omega)\right| \sim \lg \omega$ consists of three parts: (1) a horizontal part, $\omega^{\oplus}$, (2) attenuation parts, $\omega^{-1}$ and $\omega^{-2}$, and (3) two corner frequencies, $\omega_{1}$ and $\omega_{2}$. Moreover, when the length and width of the rectangular rupture plane are equal to each other, the spectral curve only includes two parts $\left(\omega^{0}\right.$ and $\left.\omega^{-2}\right)$ and one corner frequency, which is the same as the circularshear dislocation model.

\subsection{The Brune model}

The Brune model (Brune, 1970) is a semiempirical and semi-theoretical model which explains the rupture mechanisms based on stress, and which basically belongs to the circular-shear dislocation model. This model assumes that: (1) at the moment of rupture, a stress which has the same magnitude as the released stress but is along an opposite direction, is applied on the entire dislocation circle; (2) the S-wave energy takes up $80 \%$ of the total seismic-wave energy. Its far-field spectrum is:

$$
\hat{u}_{\beta}(r, \omega)=\Re_{\beta} \cdot \frac{a}{r} \cdot \frac{\Delta \sigma \cdot \beta}{\mu} \cdot \frac{1}{\omega^{2}+\omega_{c}^{2}}
$$

where $\omega_{c}$ is the corner frequency, which is the intersection frequency of the high-frequency asymptote and the low-frequency asymptote. Strictly, the Brune model is only applicable to $\mathrm{S}$-waves, but in practice this model is also used for P-waves.

\subsection{The spherical explosive source model}

The spherical explosive source model (Zhu et al., 1977) is a virtual model and is commonly 


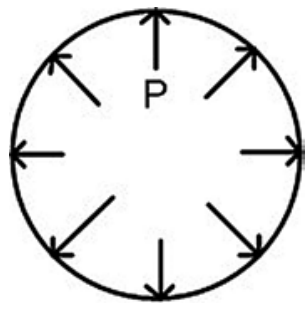

Fig. 11. A spherical explosive source.

applied because it is simple and understandable. This model assumes that: (1) the hypocentre is a spherical cavity; (2) a pressure, $\mathrm{P}_{1}$, is evenly distributed along the inner surface of the cavity which suddenly drops to $\mathrm{P}_{0}$ at time $t=0$; (3) the medium is a Poisson body and $\lambda$ $=\mu$. The spherical explosive source model is applicable to P-waves of small and medium earthquakes. The far-field radiation of the Pwave is:

$$
\begin{gathered}
u_{\alpha}(r, t)=\frac{\Delta \sigma \cdot a^{2}}{2 \sqrt{2} \mu r} \cdot e^{-W\left[t-\frac{r-a}{\alpha}\right] / \sqrt{2}} . \\
\cdot \sin W\left[t-\frac{r-a}{\alpha}\right]
\end{gathered}
$$

where $W=\frac{2 \sqrt{2} \alpha}{3 a}$. Its far-field spectrum is:

$$
\begin{aligned}
& \hat{u}_{\alpha}(r, \omega)=\frac{\Delta \sigma \cdot a^{3}}{8 \pi \mu(i \omega)}\left(\frac{1}{r^{2}}+\frac{i \omega}{\alpha r}\right) . \\
& \cdot e^{\frac{-i \omega(r-a)}{\alpha}} /\left[1+i \frac{\omega}{\omega_{0}}-\frac{3}{4}\left(\frac{\omega}{\omega_{0}}\right)^{2}\right]
\end{aligned}
$$

where $\omega_{0}=\mathrm{a} / \mathrm{a}$.

\section{Conclusions}

Common kinematic source models that are commonly used for explaining the earthquake focal mechanism, the formation of a tectonic stress field, and rupture mechanisms are presented. These models include single- and double-force-couple point-source models, a circular-shear dislocation model, a finite moving-source model, the Brune model, and the spherical explosive source model. The underlying assumptions and the applicability of each model are also discussed. Besides that, the classical elastodynamic dislocation theory, equations of motion and their solutions are presented, indicating the correlation between the displacement radiation of shear dislocation and the seismic-wave radiation. It is concluded that the models, approaches, and examples discussed here can be widely used for studies in seismology and earthquake prediction.

\section{References}

Aki, K., 1972. Earthquake mechanism. Tectonophysics 13, 421-466.

Aki, K. \& Richards, P.G., 1980. Quantitative Seismology: Theory and Methods. W.H. Freeman, San Francisco, 557 and 993 pp.

Ben-Menahem, A., 1961. Radiation of seismic surfacewaves from finite moving sources. Bulletin of the Seismological Society of America 51, 401-435.

Brune, J.N., 1970. Tectonic stress and the spectra of seismic shear waves from earthquakes. Journal of Geophysical Research 75, 4997-5009.

Burridge, R. \& Knopoff, L., 1964. Body force equivalents for seismic dislocations. Bulletin of the Seismological Society of America 54, 1875-1888.

Byerlee, J.D., 1970. Static and kinetic friction of granite at high normal stress. International Journal of Rock Mechanics and Mining Science \& Geomechanics, Abstracts 7, 577-582.

Byerly, P., 1928. The nature of the first motion in the Chilean earthquake of November 11, 1922. American Journal of Science, Series 5, 16, 232-236.

Chen, Y.-T., Lin, B.-H., Li, X.-C., Wang, M.-Y., Xia, D.-D., Wang, X.-H., Liu, W.-Q. \& Li, Z.-Y., 1976. Determination of the focus parameters of the small earthquakes in Qiaojia, Shimian, and the assessment of seismic hazard. Chinese Journal of Geophysics 19, 206-231.

Haskell, N., 1966. Total nergy and energy spectral density of elastic wave radiation from propagating faults, II, A statistical source model. Bulletin of the Seismological Society of America 56, 125-140.

Honda, H., 1957. The mechanism of the earthquakes. Science Report, Tohoku University, Series 5, Geophysics 9, Supplement, 1-46.

Kawasumi, H., 1937. An historical sketch of the development of knowledge concerning the initial motion of an earthquake. Publications du Bureau Central Seismologique International, series A, Travaux Scientifique 15, 1-76.

Li, Q.-Z., 1974. A method of studying mechanism of small earthquakes. Chinese Journal of Geophysics 17, 1-14. 
Li, Q.-Z., Wang, Z.-G., Jia, Y.-N. \& Jin, Y.-M., 1973. Stress fields of two zones obtained from single station data of small earthquakes. Chinese Journal of Geophysics 16, 23-34.

Lin, B.-H., Chen, Y.-T., Wei, F.-S. \& Li, Z.-Y., 1979. Research of asymmetric bilateral rupture process and its application in Haicheng earthquake. Acta Seismologica Sinica 1, 17-26.

McKenzie, D.P., 1969. The relation between fault plane solutions for earthquakes and the directions of the principal stresses. Bulletin of the Seismological Society of America 59, 591-601.

Reid, H.F., 1990. The mechanics of the earthquake - The California warthquake of April 18, 1906. Report of the State Investigation Commission, Vol. 2. Carnegie Institution of Washington, Washington D.C.
Savage, J.C., 1965. The effect of rupture velocity upon seismic first motions. Bulletin of the Seismological Society of America 55, 263-275.

Wyss, M. \& Brune, J.N., 1967. The Alaska earthquake of 28 March 1964: A complex multiple rupture. Bulletin of the Seismological Society of America 57, 1017-1023.

Zhu, C.-Z., Fu, C.-H., Rong, Z.-G. \& Luo, S.-L., 1977. Hypocentral parameters and quality factor of medium of small earthquakes before and after Haicheng earthquake. Acta Geophysica Sinica 20, 21-31.

Manuscript received: 28 June 2012 Revision accepted: 15 August 2012 